\title{
Grocery store interventions to change food purchasing behaviours: a systematic review of randomised controlled trials
}

\author{
C. Piernas, J. Hartmann-Boyce, F. Bianchi, S. Payne Riches, K. Frie, R. Nourse and S.A. Jebb \\ Health Behaviours, Nuffield Department of Primary Care Health Sciences, University of Oxford, \\ Radcliffe Primary Care, Oxford, UK.
}

Diet is an important determinant of health, and food purchasing is a key antecedent to consumption. Interventions in grocery stores are of interest to those trying to change food purchasing to promote health and those concerned with the marketing and sales of foods and drinks ${ }^{(1)}$

We set out to evaluate the effectiveness of grocery store interventions to change food purchasing, and to examine whether effectiveness varied based on intervention components, setting, or socioeconomic status (SES).

We conducted a systematic review of randomised controlled trials (search 6/2017). Studies must have: aimed to change food purchasing; been implemented in grocery stores (real or simulated); reported purchasing; and had a minimal control or compared interventions fulfilling our criteria. Searching, screening, bias assessment and data extraction followed Cochrane methods ${ }^{(2)}$. We grouped studies by intervention type (economic, environmental, swaps, and/or education) and synthesised results narratively. We also conducted an exploratory qualitative comparative analysis (QCA) to identify combinations of intervention components associated with statistically significant changes $(p<0.05)$ in the desired direction for at least one of the foods targeted by the intervention ${ }^{(3)}$.

We included 35 studies representing 89 interventions, $>20,000$ participants, and $>800$ stores. Risk of bias was mixed. Economic interventions showed the most promise, with eight of the nine studies in real stores and all six in simulated environments detecting an effect on purchasing. The effects of these interventions appeared to be enhanced by additional promotional activity. Swap interventions appeared promising in the two studies based in real stores. Store environment interventions showed mixed effects. Education-only interventions appeared effective in simulated environments but not in real stores. Limited data available suggested that effects of economic interventions did not differ by SES, whereas for other interventions impact was mixed. In our QCA, economic interventions (regardless of setting) and environmental and swap interventions in real stores were associated with statistically significant changes in purchasing in the desired direction for at least one of the foods targeted by the intervention, while education-only interventions in real stores were not.

Findings suggest interventions implemented in grocery stores - particularly ones which manipulate price, suggest swaps, and perhaps manipulate item availability - have an impact on purchasing and could play a role in public health strategies to improve health. Overall the evidence base for interventions in grocery stores is still limited and there is a need for more high quality studies in real grocery store settings. (https://www.crd.york.ac.uk/PROSPERO/; CRD42017068809).

1. Drewnowski A, Rehm CD (2013) Energy intakes of US children and adults by food purchase location and by specific food source. Nutrition Journal 12(1), 59.

2. Higgins JPT, Green S (2011) Cochrane Handbook for Systematic Reviews of Interventions Version 5.1.0 [updated March 2011] The Cochrane Collaboration, Available from wwwcochrane-handbookorg.

3. Thomas J, O'Mara-Eves A, Brunton G (2014) Using qualitative comparative analysis (QCA) in systematic reviews of complex interventions: a worked example. Systematic reviews 3,67 . 